\title{
Alterações sexuais no climatério do ponto de vista cinesiológico-funcional: revisão
}

\author{
Sexual changes during climacterium under a \\ kinesiological-functional approach: review
}

\author{
Sabrina Narcizo Camilo', Carolina Lazzarim De Conto², \\ Erica Feio Carneiro Nunes ${ }^{3}$, Gustavo F. Sutter Latorre ${ }^{4}$ \\ 'Faculdade Inspirar. Florianópolis, Santa Catarina, Brasil. ORCID: 0000-0002-2968-2186. sabrina_camilllo@hotmail.com \\ 2Faculdade Inspirar, Florianópolis, Santa Catarina, Brasil. ORCID: 0000-0002-3234-5543. carolina.Ic@hotmail.com.br \\ 3Universidade do Estado do Pará. Belém, Pará, Brasil. ORCID: 0000-0002-1274-4686. erica@perineo.net \\ ${ }^{4}$ Autor para correspondência. Faculdade inspirar, Florianópolis, Santa Catarina, Brasil. ORCID: 0000-0001-9806-9572. gustavo@perineo.net
}

RESUMO | INTRODUÇÃO: As mudanças físicas e psicológicas que a muIher enfrenta durante o climatério, podem prejudicar a sexualidade devido às alterações hormonais do período. OBJETIVOS: Com isso, o objetivo desta revisão é de investigar os possíveis recursos fisioterapêuticos usados sob um prisma cinesiológico funcional para minimizar os efeitos deste período. MÉTODOS: Uma busca eletrônica das seguintes bases de dados foi realizada: PubMed, Lilacs e Google Scholar. A pesquisa foi executada do ano de 2010 até o ano de 2018, utilizando as seguintes combinações de palavras: disfunção sexual feminina AND menopausa AND fisioterapia, com os termos em português e inglês. RESULTADOS: Durante o climatério ocorre uma atrofia vaginal, e essa condição pode levar a dores durante o intercurso sexual que, por sua vez, podem gerar contratura no músculo do assoalho pélvico (MAP). Uma vez instaurada esta contratura a dor tende a aumentar, assim originando um ciclo vicioso de dor. A MAP durante o climatério também sofrem uma queda da sua função muscular, podendo gerar disfunções pélvicas como a incontinência urinária, os prolapsos de órgãos pélvicos e prejudicar a função sexual da mulher. CONCLUSÕES: A fisioterapia tem um grande arsenal de recursos terapêuticos que podem incrementar a musculatura pélvica e melhorar a qualidade de vida nesse período de declínio hormonal. Apesar de se necessitar de mais estudos a esse respeito, as técnicas fisioterapêuticas que obtiveram maior eficácia no tratamento da dispareunia em mulheres no climatério, descritas nesta revisão, foram a termoterapia na MAP, liberação manual dos ponto-gatilhos miofasciais da MAP e treinamento dessa musculatura. Sendo assim, a fisioterapia pélvica deve ser uma linha terapêutica a ser prescrita no climatério.

PALAVRAS-CHAVE: Fisioterapia. Menopausa. Assoalho pélvico. Disfunções sexuais fisiológicas.
ABSTRACT | INTRODUCTION: The physical and psychological changes that the woman faces during the climacteric can damage sexuality due to the hormonal changes of the period. OBJECTIVES: Therefore, the objective of this review is to investigate the possible physiotherapeutic resources used under a functional kinesiological prism to minimize the effects of this period. METHODS: An electronic search of the following databases was performed: PubMed, Lilacs and Google Scholar. The research was performed from the year 2010 to the year 2018, using the following combinations of words: female sexual dysfunction AND menopause AND physiotherapy, with the terms in Portuguese and English. RESULTS: During climacteric vaginal atrophy occurs, and this condition can lead to pain during sexual intercourse which, in turn, may lead to contractures in the pelvic floor muscles (PFM), once this contracture is established, the pain tends to increase, thus giving rise to a vicious cycle of pain. PFMs during the climacteric also suffer a decrease of their muscular function, being able to generate pelvic dysfunctions, such as urinary incontinence, prolapses of pelvic organs and also to impair the sexual function of the woman. CONCLUSIONS: With this, physiotherapy has a great arsenal of therapeutic resources that can boost pelvic floor muscles and improve the quality of life in this period of hormonal decline. Despite the need for further studies in this regard, the physiotherapeutic techniques that were most effective in the treatment of dyspareunia in menopausal women, described in this review, were the thermotherapy in PFM, manual release of PFM myofascial trigger points and training of this musculature. Therefore, pelvic physiotherapy should be a therapeutic line to be prescribed in climacteric.

KEYWORDS: Physiotherapy. Menopause. Sexual dysfunction, Physiologic. Pelvic floor. 


\section{Introdução}

As mudanças corporais durante o climatério podem refletir na vida da mulher de diversas formas. Sabese que o déficit hormonal do estrogênio pode resultar em disfunções ${ }^{1}$ como irregularidades menstruais, distúrbios do sono, sintomas vasomotores e alterações urogenitais².

Estima-se que de $10 \%$ a $50 \%$ de mulheres na pósmenopausa apresentam sinais e sintomas de atrofia urogenital ${ }^{3,4}$. Esses sintomas incluem ressecamento, irritação, prurido e dispareunia ${ }^{3}$, também podendo estar associadas a disfunções miccionais, evacuatórias, culminando em prejuízos psicológicos e emocionais, com impacto importante na qualidade de vida'.

As alterações no epitélio vaginal e na musculatura do assoalho pélvico (MAP), ambas por conta de alterações hormonais nas mulheres no climatério, são fatores de importância no comprometimento da atividade sexual feminina nesse período ${ }^{2,5}$. O assoalho pélvico é uma unidade musculoesquelética que fornece suporte e controle aos órgãos pélvicos e suas respectivas funções. Sua anatomia e funcionalidade são a base de funções fisiológicas básicas como o armazenamento e evacuação de urina e fezes, suporte de órgãos pélvicos e a função sexual em si ${ }^{6}$. Modernamente o comprometimento cinesiológico-funcional destas funções tem recebido destaque na literatura. Particularmente, a função sexual e seus comprometimentos têm no componente musculoesquelético e funcional forte candidato à etiologia das disfunções ${ }^{7,8}$.
Todavia parece ainda não haver consenso na literatura a respeito das alterações sexuais das muIheres no climatério, especialmente quando estudadas sob o prisma cinesiológico funcional, sendo este, por fim, o objetivo da presente revisão.

\section{Metodologia}

Foi realizada uma busca eletrônica nas bases de dados PubMed, Lilacs e Google Scholar, restrita a ensaios clínicos publicados de 2010 até 2018 , utilizando as seguintes combinações de palavras: disfunção sexual feminina AND climacterium AND fisioterapia, incluindo suas variantes em inglês. A busca foi repetida para verificar os resultados.

As revisoras avaliaram os títulos e resumos dos artigos identificados na busca inicial para verificar os critérios de inclusão e exclusão. Foram adotados como critérios de inclusão o estado de menopausa; presença de disfunção sexual e tratamento por fisioterapia. Os estudos não-incluídos foram estudos de caso, ensaios em cobaias e artigos duplicados nas bases de dados.

Caso o título e o resumo do artigo não apresentassem nitidamente se $o$ artigo poderia ser incluso, 0 mesmo era lido na integra e classificado.

As informações dos estudos resultantes foram extraídas de maneira descritiva, de acordo com as características da população e o tipo de intervenção fisioterapêutica utilizada. Foram analisadas as disfunções sexuais e as medidas de intervenção, expondo quais foram utilizadas e a maneira de análise em cada uma delas. 


\section{Resultados}

A busca eletrônica, contabilizando as palavras-chaves, gerou um total de 984 artigos. Após a seleção dos artigos perante os critérios de inclusão, 8 artigos foram selecionados como estudos potenciais com base no título e no resumo. Os não-incluídos no estudo foram 977 artigos por não estarem na língua portuguesa ou inglesa, estudos com homens ou eram revisões. A maioria absoluta das não inclusões veio da base Google Scholar que, por definição, apresenta também cartas editoriais, artigos de síntese, respostas, dissertações e teses, comentários, etc. Todos os artigos foram lidos na integra, sendo que 5 foram excluídos, pois os estudos tratavam somente de avaliação e não de intervenção clínica (Figura 1).

Figura 1. Fluxograma da seleção dos artigos primários
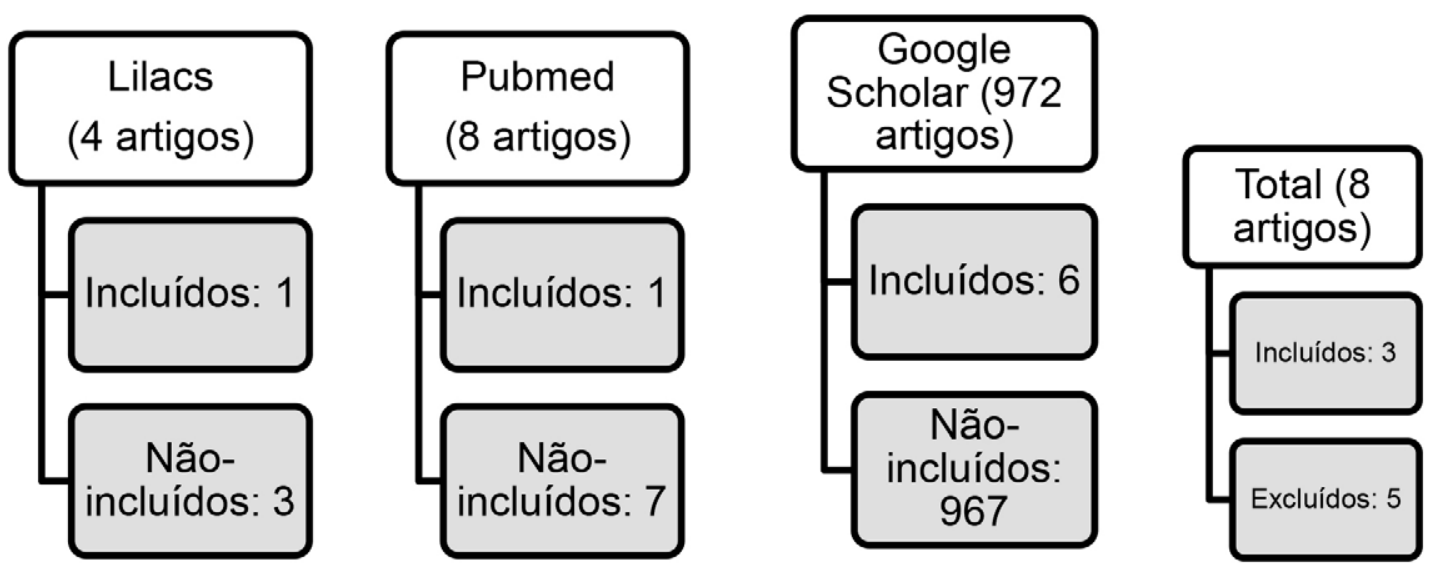

A Tabela 1 caracteriza a população dos 3 artigos selecionados que contém informações sobre os estudos e estão dispostos pelo nome do autor e o ano, os métodos utilizados, os resultados e a conclusão.

Lara et al. ${ }^{9}$ realizaram um estudo com 32 mulheres sexualmente ativas na pós-menopausa, realizaram o TMAP e exercícios de aquecimento e alongamentos, em sessões inidividuais, duas vezes por semana por 12 semanas. Para avaliação foram utilizados os questionários Quociente Sexual Feminino (QS-F) e a Escala de Oxford. O escore médio da escala de Oxford após o protocolo foi significantemente maior do que a pontuação antes do protocolo. Como resultados os autores observaram que mulheres na pós-menopausa, sedentárias e continentes, quando submetidas a um programa de exercícios que inclua o TMAP, melhoraram efetivamente a ansiedade e a força da MAP, todavia, não houve impacto significativo sobre a função sexual medida pelo SQ-F99.

Lisboa et al. ${ }^{10}$ analisaram dois grupos de mulheres na pós-menopausa, divididas em dois grupos: fibromialgia ( $n=47$ ) e controle $(n=43)$. Foram utilizados os questionários Utian Quality of Life (UQOL), Índice Menopausal de Blatt-Kupperman (IMBK) e o QS-F. O estudo consistia num protocolo de 10 semanas de tratamento feitas duas vezes por semana, com duração diária de uma hora, em sessões individuais. A conduta proposta obedeceu à sequência de percepção, dissociação abdominoperineal, contração voluntária e automatização do assoalho pélvico. Os resultados nos domínios de qualidade de vida apresentaram melhoria estatisticamente significativa para ambos os grupos. Na avaliação intergrupos pós-tratamento foi observada diferença estatisticamente significante em três dos quatro domínios do UQOL (ocupacional, saúde e sexual), sendo que a função sexual teve seus escores melhorados em ambos grupos, mas esta foi ainda mais evidente no grupo controle. A sintomatologia climatérica apresentou redução significativa para ambos os grupos. Assim, conclui-se que a cinesioterapia para o assoalho pélvico proporciona melhorias significativas na qualidade de vida, nos sinais e sintomas climatéricos e na função sexual, todavia os sintomas da fibromialgia impactam negativamente sobre a evolução do TMAP em mulheres no climatério ${ }^{10}$. 
Schvartzman et al. ${ }^{11}$, através de um ensaio clínico randomizado utilizando os questionários Índice da Função Sexual Feminina (FSFI), Escala Cervantes, Escala Visual da dor e a avaliação da MAP através de eletromiografia e palpação vaginal através da Escala New Perfect, objetivaram verificar se 42 mulheres no climatério e com dispareunia melhorariam seus sintomas com um protocolo de fisioterapia pélvica. No grupo intervenção foi aplicada a termoterapia e liberação manual dos pontos gatilhos nos músculos do assoalho pélvico e TMAP individual durante cinco sessões. No grupo controle foi aplicada a termoterapia na região lombar e realizada a liberação manual das musculaturas do diafragma abdominal, piriforme, e iliopsoas, sem envolvimento da MAP. Os resultados foram satisfatórios, uma vez que os escores de dor no grupo intervenção diminuíram de média 7,77 para 2,25 na escala EVA, e no grupo controle, de média 7,62 para 5,58. Com isso pode-se dizer que a intervenção realizada diretamente na MAP foi associada a uma redução estatisticamente significante nos escores de dor. Verificou-se também melhora nos escores da Escala New Perfect, nos escoes totais do FSFI e da Escala Cervantes ${ }^{11}$.

Dentre os três artigos, o estudo de Schvartzman et al. ${ }^{11}$ obteve melhores resultados sobre a função sexual, uma vez que a escala análogica visual de dor diminuiu significativamente em ambos os grupos, e os escores gerais do FSFI aumentaram. Sendo assim, o protocolo de fisioterapia descrito desempenhou uma melhora significativa em mulheres climatéricas com dispareunia.

Tabela 1. Caracterização da população dos estudos

\begin{tabular}{|c|c|c|c|}
\hline Autor/Ano & Métodos & Resultados & Conclusão \\
\hline Lara et al. $2012^{9}$ & $\begin{array}{l}\text { Estudo prospectivo, longitudinal e exploratório. } \\
\text { Mulheres sexualmente ativas na pós-menopausa ( } \mathrm{n}= \\
\text { 32) } \\
\text { Questionários: SQ-F e HADS; } \\
\text { Avaliação MAP: palpação bi manual vaginal e a força } \\
\text { muscular pela escala de Oxford Modificada. } \\
\text { Protocolo: Individual ou grupo? TMAP e exercícios de } \\
\text { aquecimento e alongamentos. Duas vezes por semana } \\
\text { por } 12 \text { semanas. }\end{array}$ & $\begin{array}{l}\text { Aumento do escore na escala de Oxford após o } \\
\text { protocolo. } \\
\text { O número de mulheres que sofreram ansiedade após } \\
\text { o protocolo foi significativamente menor. } \\
\text { Não houve mudança na proporção de mulheres com } \\
\text { depressão e na pontuação geral do SQ-F. }\end{array}$ & $\begin{array}{l}\text { O presente estudo de mulheres na pós-menopausa } \\
\text { sedentários e continente indica que a implementação de um } \\
\text { programa de exercícios que inclua o TMAP melhora } \\
\text { efetivamente a ansiedade e a força dos MAP, mas não teve } \\
\text { impacto na função sexual medida pelo SQ-F. }\end{array}$ \\
\hline Lisboa et al. $2015^{10}$ & $\begin{array}{l}\text { Ensaio clínico. } \\
\text { Mulheres na pós-menopausa. } \\
\text { As voluntárias foram divididas em dois grupos: } \\
\text { fibromialgia ( } n=47 \text { ) e controle ( } n=43 \text { ). } \\
\text { Questionários: UQOL, IMBK e o QS-F. } \\
\text { Protocolo: Cinesioterapia de AP por } 10 \text { semanas feitas } \\
\text { duas vezes por semana, com duração diária de uma } \\
\text { hora. Individual ou grupo? }\end{array}$ & $\begin{array}{l}\text { Os domínios de qualidade de vida tiveram uma } \\
\text { melhoria estatisticamente significante em ambos os } \\
\text { grupos. } \\
\text { A função sexual teve seus escores elevados em } \\
\text { ambos os grupos, mas foi evidenciado melhora mais } \\
\text { significante no grupo controle. } \\
\text { A sintomatologia climatérica teve redução significante } \\
\text { nos escores para ambos os grupos. }\end{array}$ & $\begin{array}{l}\text { Conclui-se no presente estudo que a cinesioterapia para o } \\
\text { assoalho pélvico proporciona melhorias significantes na } \\
\text { qualidade de vida, sinais e sintomas climatéricos e na } \\
\text { função sexual. }\end{array}$ \\
\hline $\begin{array}{l}\text { Schvartzman et al., } \\
2016^{11}\end{array}$ & $\begin{array}{l}\text { Ensaio clínico randomizado. } \\
\text { Questionários: FSFI, Escala Cervantes, Escala Visual da } \\
\text { dor. } \\
\text { Avaliação MAP: Eletromiografia e Escala New Perfect. } \\
\text { Foram incluídas no estudo } 42 \text { mulheres climatéricas } \\
\text { com dispareunia que foram divididas em dois grupos. } \\
\text { O protocolo consistia em termoterapia, cinesioterapia } \\
\text { e TMAP }\end{array}$ & $\begin{array}{l}\text { Os escores de dor no grupo intervenção e controle } \\
\text { tiveram uma diminuição estatisticamente significante. }\end{array}$ & $\begin{array}{l}\text { O protocolo de fisioterapia proposto foi eficaz para a } \\
\text { melhora da dispareunia, da qualidade de vida, da função } \\
\text { sexual e da funcionalidade da MAP em mulheres } \\
\text { climatéricas com dispareunia. }\end{array}$ \\
\hline
\end{tabular}

\section{Discussão}

O presente estudo teve como objetivo apontar as alterações sexuais em mulheres no climatério, analisadas sob a ótica cinesiológica-funcional. Primeiramente destaca-se o baixo número de artigos que se enquadraram nos critérios de inclusão, mesmo ante a importância do tema.

Dentre os artigos selecionados, todos avaliaram a MAP, a função sexual e a qualidade de vida das mulheres no climatério, observando a importância e a ligação dos aspectos físicos e psicológicos, principalmente nesse período, uma vez que se sabe do declínio da função sexual e da qualidade de vida na menopausa. A população dos três estudos incluídos não foi homogênea, o que dificultou a comparação dos resultados.

Durante o climatério ocorre uma atrofia vaginal em conjunto com a redução da lubrificação vaginal, devido ao decréscimo hormonal, e essa condição pode levar as dores durante o intercurso sexual que, por sua vez, podem gerar contratura na MAP ${ }^{12,13}$. Uma vez instaurada esta contratura a dor tende a aumentar, e assim originando um ciclo vicioso de dor. A MAP durante o climatério também sofrem uma queda da sua função muscular, devido 
a uma diminuição da espessura epitelial vaginal, da densidade da camada do músculo liso, dos vasos sanguíneos, da morfologia e densidade das terminações nervosas e da estrutura do colágeno, podendo gerar disfunções pélvicas, como a incontinência urinária, os prolapsos de órgãos pélvicos e também prejudicar a função sexual da mulher ${ }^{14,15,16}$. O treinamento dos músculos do assoalho pélvico para essa população gera benefícios para as disfunções já instauradas ou como modo preventivo, dando mais percepção muscular, alivio das dores e coordenação muscular, obtendo uma melhora física e psicológica ${ }^{17}$.

É escassa também a evidência a respeito dos protocolos de tratamento para a disfunção sexual feminina, e esta observação foi corroborada pela variedade de protocolos descrita nos três estudos que compuseram a presente revisão. Lara et al. ${ }^{9}$ utilizaram um protocolo de 12 semanas realizado duas vezes por semana, consistindo de quatro séries de dez repetições de contrações máximas do assoalho pélvico mantidos por seis segundos, seguido por três contrações rápidas de um segundo cada, nas posições supina, sentada, mãos no joelho e em pé. O programa também incluiu exercícios de aquecimento, alongamento muscular para o quadríceps, isquiotibiais, tríceps sural, paravertebrais, abdominais e MAP, e relaxamento ${ }^{9}$.

Já Lisboa et al. ${ }^{10}$ apresentaram um protocolo executado em 10 semanas consecutivas e envolveu 20 sessões de cinesioterapia para a MAP, feitas duas vezes por semana, com duração diária de uma hora. A conduta proposta seguiu a sequência de percepção, dissociação entre musculatura abdominal e a do assoalho pélvico, contração voluntária e automatização do assoalho pélvico associado a posturas facilitadoras, mobilização de pelve e treino respiratório no momento das contrações dos MAP'10.

Por fim, Schvartzman et al. ${ }^{11}$ aplicaram termoterapia na MAP, liberação manual dos ponto-gatilhos miofasciais da MAP e treinamento dessa musculatura durante cinco sessões. No grupo controle foi aplicada a termoterapia na região lombar e realizada a liberação manual miofascial das musculaturas do diafragma abdominal, piriforme, e iliopsoas, sem envolvimento da MAP ${ }^{11}$.

Percebe-se que nenhum dos artigos utilizou recursos terapêuticos de eletroestimulação para analgesia como, o TENS, obtendo melhora na dor somente com as técnicas manuais utilizadas.
O estudo de Lara et al. ${ }^{9}$ teve a idade média de 52 anos, todas as mulheres estavam em longos relacionamentos. O escore médio da escala de Oxford após o protocolo foi significativamente maior do que a pontuação antes do protocolo. O número de mulheres que sofreram ansiedade após o protocolo foi significativamente menor do que antes do protocolo, contudo, não houve mudança significativa na proporção de mulheres com depressão e na pontuação geral do SQ-F após o tratamento. A depressão é um fator conhecidamente presente no período da menopausa, e está relacionada tanto a fatores físicos quanto emocionais ${ }^{18}$. Desse modo, o fato de que Lara et al. ${ }^{9}$ não obtiveram resultados satisfatórios quanto a melhoria da depressão das pacientes deve ser interpretado com cautela, pois pode não necessariamente significar que exercícios para o assoaIho pélvico no climatério não auxiliem no combate à depressão destas mulheres, mas possa depender de um protocolo de exercícios com dosagem, por exemplo, mais agressiva. Ainda, é importante ressaltar que a depressão neste período pode não estar relacionada unicamente aos problemas sexuais, mas também a outros fatores gerais do envelhecimento, para os quais a fisioterapia específica do assoalho pélvico pode não alcançar. Essas intrigantes questões certamente merecem maior estudo.

Já o estudo de Lisboa et al. ${ }^{10}$ com 83 participantes, das quais 43 faziam parte do grupo fibromialgia e 40 do grupo controle, mostrou que o protocolo cinesioterapêutico trabalhado possibilitou melhoria na qualidade de vida tanto para o grupo de fibromialgia como para o grupo controle, em todos os domínios. Na avaliação da função sexual, observa-se que, após a intervenção, tanto o grupo fibromialgia como o controle elevaram seus escores, com diferenças estatisticamente significantes. De fato, o treinamento do assoalho pélvico melhora significativamente a função sexual de mulheres com todo tipo de queixas sexuais $^{19}$, e o estudo de Lisboa e colaboradores demonstra que este treinamento é também eficaz na melhoria da função sexual de mulheres na menopausa, e também de mulheres na menopausa vitimadas ainda pela fibromialgia - embora os resultados neste último grupo tenham sido ligeiramente mais modestos que no grupo sem a fibromialgia. Parece possível que o treinamento da musculatura do assoalho pélvico melhore a função sexual inclusive de mulheres saudáveis, tema que também merece melhor investigação considerando a melhoria da função sexual significa melhoria na qualidade vida em geral ${ }^{19}$. 
Schvartzman et al. ${ }^{11}$, demonstraram que os exercícios para a MAP corresponderam a uma redução estatisticamente significativa nos escores de dor sexual, função do assoalho pélvico e da função sexual em geral. De fato, a fisioterapia pélvica é eficiente no tratamento da dor sexual ${ }^{8,20}$, função do assoalho pélvico ${ }^{7,19,20,21} \mathrm{e}$ na melhoria da função sexual feminina em geral7 7,819,20.

Os exercícios do assoalho pélvico auxiliam na melhoria da consciência corporal e fortalecimento da MAP, que atuam como estabilizadora dos órgãos pélvicos, como útero e bexiga, agem na continência urinária e fecal e podem contribuir nas distintas fases da resposta sexual feminina como desejo, excitação e orgasmo ${ }^{20}$.

Embora ainda haja poucos estudos sobre a fisioterapia pélvica sobre a função sexual feminina, seus recursos podem ser utilizados como modalidades sem contraindicações ou riscos em potencial ${ }^{21}$, podendo ser úteis inclusive no tratamento da disfunção sexual em mulheres após a menopausa.

\section{Conclusão}

A menopausa é um período natural do ciclo de vida da mulher e pode gerar prejuízos em todos os aspectos da vida, como físico, social e psicológico. Dentro dos aspectos físicos podemos destacar as disfunções sexuais, como a falta de desejo, a baixa lubrificação e a dispareunia. Por se tratarem de problemas físicos e funcionais a fisioterapia possui arsenal de recursos terapêuticos que, ao melhorarem a função da musculatura do assoalho pélvico e a sensibilidade local, auxiliam a melhora da qualidade de vida mesmo nesse período de declínio hormonal. Apesar de se necessitar de mais estudos a esse respeito, as técnicas fisioterapêuticas que obtiveram maior eficácia no tratamento da dispareunia em mulheres na menopausa, descritas nesta revisão, foram a termoterapia na MAP, liberação manual dos ponto-gatilhos miofasciais da MAP e o treinamento dessa musculatura, de modo que a fisioterapia pélvica deve ser uma linha terapêutica a ser prescrita no climatério.

\section{Contribuições dos autores}

Camilo SN e Conto CL responsabilizaram-se pela redação do artigo científico. Latorre GFS participou da concepção, delineamento e interpretação dos resultados. Nunes EFC revisou criticamente o conteúdo do artigo.

\section{Conflitos de interesses}

Nenhum conflito financeiro, legal ou político envolvendo terceiros (governo, empresas e fundações privadas, etc.) foi declarado para nenhum aspecto do trabalho submetido (incluindo mas não limitando-se a subvenções e financiamentos, participação em conselho consultivo, desenho de estudo, preparação de manuscrito, análise estatística, etc.).

\section{Referências}

1. Etienne MA, Waitman MC. Disfunções Sexuais Femininas. São Paulo: LMP; 2006. p.178.

2. Bachmann G, Leiblum SR. The impact of hormones on menopausal sexuality: a literature review. Menopause J North Am Menopause Soc. 2004;11(1):120-30. doi: 10.1097/01. GME.0000075502.60230.28

3. Parish SJ, Nappi RE, Krychman ML, Kellogg-Spadt S, Simon JA, Goldstein JA et al. Impact of vulvovaginal health on postmenopausal women: a review of surveys on symptoms of vulvovaginal atrophy. Int J Womens Health 2013; 5:437-47. doi: 10.2147/lJWH.S44579

4. Farage $\mathrm{M}$, Maibach $\mathrm{H}$ : Lifetime changes in the vulva and vagina. Arch Gynecol Obstet 2006;273(4):195-202. doi: 10.1007/s00404005-0079-x

5. Abdo C, Fleury H, Afif-Abdo J. Transtornos da Função Sexual. In: Busatto GF. Fisiopatologia dos transtornos psiquiátricos. São Paulo: Atheneu; 2006. p. 235-48.

6. Abrams P, Andersson KE, Birder L, Brubaker L, Cardozo L, Chapple $C$ et al. Fourth International Consultation on Incontinence Recommendations of the Scientific Committee: Evaluation and Treatment of Urinary Incontinence, Pelvic Organ Prolapse, and Fecal Incontinence. Neurourol Urodyn 2010;29:213-40. doi: 10.1002/nau.20870 
7. Bø K, Berghmans B, Mørkved S, Van Kampen M. Evidence-Based Physical Therapy for the Pelvic Floor: Bridging Science and Clinical Practice. 2. ed. London: Churchill Livingstone, 2015. p. 446.

8. Latorre GFS, Manfredini CC, Demterco PS, Barreto VMNF, Nunes EFC. A fisioterapia pélvica no tratamento da vulvodínia: revisão sistemática. Femina. 2015;43(6)257-264.

9. Lara LA, Montenegro ML, Franco MM, Abreu DC, Rosa e Silva AC, Ferreira CH. Is the Sexual Satisfaction of Postmenopausal Women Enhanced by Physical Exercise and Pelvic Floor Muscle Training? J Sex Med 2012;9(1):218-23. doi: 10.1111/j.1743$\underline{6109.2011 .02516 . x}$

10. Lisboa LL, Sonehara E, Oliveira KCAN de, Andrade SC de, Azevedo GD. Efeito da cinesioterapia na qualidade de vida, função sexual e sintomas climatéricos em mulheres com fibromialgia. Rev Bras Reumatol. 2015;55(3):209-215. doi: 10.1016/j. rbr.2014.08.019

11. Schvartzman R. Intervenção fisioterapêutica em mulheres climatéricas com dispareunia: ensaio clínico randomizado. [Tese de Doutorado]. Universidade Federal do Rio Grande do Sul, Porto Alegre; 2016.

12. Al-Baghdadi O, Ewies AA. Topical estrogen therapy in themanagement of postmenopausal vaginal atrophy: An up-to-date overview. Climacteric. 2009; 12(2):91-105. doi: 10.1080/13697130802585576

13. Mac Bride MB, Rhodes DJ, Shuster LT. Vulvovaginal atrophy. Mayo Clinic Proceedings. 2010; 85(1):87-94. doi: $10.4065 /$ mcp.2009.0413

14. Lara LA, Useche B, Rosa-e-Silva JC, Ferriani RA, Reis RM, Sa MF et al. The effects of hypoestrogenism on the vaginal wall: Interference with the normal sexual response. J Sex Med 2009;6(1):30-9. doi: 10.1111/j.1743-6109.2008.01052.x

15. Groeneveld FP, Bareman FP, Barentsen R, Dokter HJ, Drogendijk AC, Hoes AW. The climacteric and well-being. J Psychosom Obstet Gynaecol. 1993;14(2):127-43.

16. Dennerstein L, Randolph J, Taffe J, Dudley E, Burger H. Hormones, mood, sexuality, and the menopausal transition. Fertil Steril 2002;77(suppl 4):S42-8. doi: 10.1016/s0015-0282(02)03001-7

17. Zahariou AG, Karamouti MV, Papaioannou PD. Pelvic floor muscle training improves sexual function of women with stress urinary incontinence. Int Urogynecol J Pelvic Floor Dysfunct 2008;19(3):401-6. doi:10.1007/s00192-007-0452-3
18. Vivian TJ, Hickey M. Menopause and depression: is there a link? Maturitas. 2014;79(2):142-6. doi: 10.1016/j. maturitas.2014.05.014

19. PiassarollI VP, Hardy E, Andrade NF de, Ferreira N de O, Osis MJD. Treinamento dos músculos do assoalho pélvico nas disfunções sexuais femininas. Rev Bras Ginecol Obstet. 2010;32(5):234-40. doi: 10.1590/S0100-72032010000500006

20. Rosenbaum TY. Physiotherapy treatment of sexual pain disorders. J Sex Marital Ther. 2005;31(4):329-40. doi: 10.1080/00926230590950235

21. Berghmans LCM, Hendriks HJM, Bø K, Hay-Smith EJC, Bie RA, Van WVDE. Conservative treatment of stress genuine incontinence in women: a systematic review of randomized clinical trials. $\mathrm{Br}$ Jrol.1998;82(2):181-91. doi: 10.1046/j.1464-410x.1998.00730.x 